\title{
Disturbed alveolar fibrin turnover during pneumonia is restricted to the site of infection
}

\author{
G. Choi*,\#,ף, M.J. Schultz*, , J.W.O. van Till ${ }^{+}$, P. Bresser ${ }^{\S}$, J.S. van der Zee ${ }^{\S}$, M.A. Boermeester ${ }^{+}$, \\ M. Levi" ${ }^{\#}$, T. van der Poll ${ }^{\#,}$
}

Disturbed alveolar fibrin turnover during pneumonia is restricted to the site of infection. G. Choi, M.J. Schultz, J.W.O. van Till, P. Bresser, J.S. van der Zee, M.A. Boermeester, M. Levi, T. van der Poll. (C) ERS Journals Ltd 2004.

ABSTRACT: Severe infection is associated with profound alterations in the systemic haemostatic balance, with activation of coagulation and suppressed fibrinolysis. Within the alveolar compartment, similar disturbances have been described during pulmonary inflammation. The current authors investigated whether local haemostasis was influenced during ventilator-associated pneumonia (VAP).

In five patients with unilateral VAP, bronchoalveolar lavage fluid (BALF) was obtained from both the infected site (as identified on chest radiograph) and the contralateral noninfected lung (with no clinical or radiographic abnormalities). Markers for coagulation and fibrinolysis were compared between infected and noninfected lungs. A total of $\mathbf{1 0}$ healthy volunteers and $\mathbf{1 0}$ mechanically ventilated patients without pneumonia served as controls.

Strong activation of coagulation (high levels of thrombin-antithrombin complexes, soluble tissue factor and factor VIIa) was detected in BALF from infected lungs, compared with that from noninfected lungs and controls. Furthermore, in infected lungs, fibrinolysis was depressed, with high levels of plasminogen activator inhibitor type 1 .

In conclusion, ventilator-associated pneumonia is characterised by a hypercoagulant state at the site of infection.

Eur Respir J 2004; 24: 786-789.
Depts of *Intensive Care Medicine, ${ }^{\#}$ Internal Medicine, "Laboratory of Experimental Internal Medicine, ${ }^{+}$Surgery, and ${ }^{\S}$ Pulmonology, Academic Medical Centre, University of Amsterdam, Amsterdam, the Netherlands.

Correspondence: G. Choi, Academic Medical Centre, University of Amsterdam, Dept of Intensive Care Medicine, C3-423, Meibergdreef 9, 1108 AZ Amsterdam, the Netherlands. Fax: 31205669568

E-mail: GodaChoi@mail.com

Keywords: Bronchoalveolar lavage fluid coagulation

fibrinolysis

mechanical ventilation

plasma

pneumonia

Received: December 192003

Accepted after revision: June 262004
Sepsis in critically ill patients is almost invariably associated with a disturbed haemostatic balance. Increased expression of tissue factor (TF) promotes activation of the coagulation pathway; concordantly, fibrinolytic activity is suppressed, mainly by upregulation of plasminogen activator inhibitor type 1 (PAI-1), the main inhibitor of plasminogen activation [1]. The most severe clinical manifestation of these alterations is a syndrome known as disseminated intravascular coagulation, characteristically associated with intravascular deposition of fibrin [2]. Remarkably, similar alterations in fibrin turnover have been demonstrated on a local level during pulmonary bacterial infections, leading to fibrin deposits in the interstitial and alveolar spaces of the lung [1, 3-5]. The current authors recently described the clinical course of ventilator-associated pneumonia (VAP), in which procoagulant activity was increased within the pulmonary compartment and fibrinolysis was decreased [3]. Whilst this longitudinal study examined haemostatic alterations in fluid obtained from the airways in a nondirected way, in the present study, the aim was to determine whether the disturbed fibrin turnover was restricted to the site of infection. To achieve this, bilateral bronchoalveolar lavage (BAL) was performed on patients with unilateral VAP, and markers of coagulation and fibrinolysis in BAL fluid (BALF) from the infected lung were compared with that from the contralateral noninfected lung. Healthy volunteers and mechanically ventilated patients without pneumonia served as controls.

\section{Methods}

\section{Patients}

The study protocol was reviewed and approved by the Medical Ethics Committee of the University of Amsterdam. Written informed consent was obtained from all subjects or their legal representatives. Five consecutive patients with unilateral VAP were investigated, fulfilling the following criteria: fever or hypothermia $\left(<35.0\right.$ or $\left.>37.7^{\circ} \mathrm{C}\right)$; leukocytosis or leukopenia $\left(<3\right.$ or $\left.>10 \times 10^{9} \cdot \mathrm{L}^{-1}\right)$; worsening of arterial oxygen tension $\left(\mathrm{Pa}, \mathrm{O}_{2}\right) /$ inspiratory oxygen fraction $\left(\mathrm{F}, \mathrm{O}_{2}\right)$ ratios; and a novel unilateral infiltrate on chest radiograph. The diagnosis had to be supported by the results of microbiological culture of the BALF, or a clinical course consistent with VAP. For safety reasons, patients were excluded with $\mathrm{Pa}, \mathrm{O}_{2} \leqslant 10 \mathrm{kPa}$ and $\mathrm{Fi}_{1}, \mathrm{O}_{2} \leqslant 0.60$. A total of 10 mechanically ventilated patients without any signs of pulmonary infection and 10 healthy individuals (mean \pm SEM $32 \pm 8$ yrs old) served as controls.

\section{Sampling and specimen processing}

BALF was obtained in a standardised fashion, according to the guidelines of the American Thoracic Society, using a flexible fibreoptic video-bronchoscope. Seven successive 
20-mL aliquots of pre-warmed $0.9 \%$ saline were instilled in a subsegment of the lung, and each was aspirated immediately with low suction (general recovery $10-15 \mathrm{~mL}$ ). BAL was initiated at the noninfected lung in a subsegment of the middle lobe or lingula, followed by lavage of a subsegment of the infected lobe, as localised on chest radiograph. Recovery from the infected and noninfected sites did not differ. In all control subjects, BALF was obtained from the right middle lobe. Prior to lavage, blood samples were drawn into sterile vacutainer tubes containing citrate. The first aliquot was discarded; the second and third BALF recoveries from both sites were sent for microbial culture and virus isolation. In addition to the blood samples, the remaining BALF was centrifuged at $3,000 \mathrm{rpm}$ for $15 \mathrm{~min}$ at $4^{\circ} \mathrm{C}$. Supernatants were stored at $-80^{\circ} \mathrm{C}$ until assays were performed.

\section{Measurements}

Thrombin-antithrombin complex (TATc; Behringwerke AG, Marburg, Germany), soluble TF (sTF; American Diagnostics, Greenwich, CT, USA), PAI-1 (TintElize PAI-1; Biopool, Umea, Sweden), tumour necrosis factor (TNF)- $\alpha$ and interleukin (IL)-6 concentrations (Sanquin Diagnostics, Amsterdam, The Netherlands) were measured using specific commercially available ELISAs, according to the manufacturer's instructions. Levels of factor VIIa (FVIIa) were determined by an enzyme-capture assay for determining FVIIa activity, urokinase-type plasminogen activator (u-PA) levels by means of ELISA, and plasminogen activator activity (PAA) using an amidolytical assay, all as described previously [3].

\section{Statistical analysis}

Wilcoxon signed-rank test was used to compare paired BALF samples from infected and noninfected lungs. Differences between patients with VAP and controls were analysed using the Mann-Whitney U-test. BALF and plasma levels were expressed as median with interquartile range, unless otherwise stated. A p-value $\leqslant 0.05$ was considered statistically significant.

\section{Results}

Patient characteristics are presented in table 1. All patients with VAP underwent two-sided BAL within $12 \mathrm{~h}$ of fulfilling the inclusion criteria, and recovered uneventfully with antibiotic therapy. All patients received low-molecular heparin (deep venous thrombosis-prophylaxis). No other anticoagulant medication was given. Mechanically ventilated controls, matched in age, ventilation duration and mechanical ventilator settings, showed no signs of pulmonary infection throughout their clinical course. Mechanical ventilation settings were as follows. In all patients, tidal volumes were maintained as $6-8 \mathrm{~mL} \cdot \mathrm{kg}^{-1}$ using a pressure-controlled or pressure-support mode. Positive end-expiratory pressure (PEEP) levels were set according to a strict protocol, in which optimal PEEP was defined as the lowest level of PEEP with maximum $\mathrm{Pa}, \mathrm{O}_{2}$. Levels of PEEP were 9.0 (8.0-11.5) $\mathrm{cmH}_{2} \mathrm{O}$ in patients with VAP, and $8.0(7.3-13.0) \mathrm{cmH}_{2} \mathrm{O}$ in mechanically ventilated controls $(\mathrm{p}=0.9)$.

BALF levels of coagulation and fibrinolysis activity are presented in figure 1. In VAP patients, levels of TATc, STF and FVIIa were significantly higher in BALF from the infected lung, as compared with BALF levels from the contralateral noninfected lung. Levels of coagulation in the noninfected lungs were higher than in BALF from control subjects. In VAP patients, PAA was significantly lower in infected lungs, whilst concentrations of PAI-1 and u-PA were significantly higher. In noninfected lungs from VAP patients, PAA was lower than in BALF from control subjects. PAI-1 and u-PA concentrations were significantly lower in controls, as compared with levels from noninfected lungs in VAP patients.

In plasma, TATc levels were similar in patients with VAP and mechanically ventilated controls $(9.2$ (8.0-9.8) and 7.1 (6.3-9.2) $\mathrm{mU} \cdot \mathrm{mL}^{-1}$, respectively), but levels were significantly higher than in healthy subjects $\left(4.7(3.0-5.2) \mathrm{mU} \cdot \mathrm{mL}^{-1}\right.$, both $\mathrm{p}<0.01)$. Plasma levels of PAA were equal in all groups (median 101\%).

Concentrations of TNF- $\alpha$ were very low or undetectable in BALF from all patients and healthy subjects (no difference between groups). IL-6 levels in BALF showed a large variation; the highest concentrations were found in BALF from infected lungs $\left(574(102-1399) \mathrm{pg} \cdot \mathrm{mL}^{-1}\right)$. In BALF from noninfected lungs, levels were $83.4(20.1-559) \mathrm{pg} \cdot \mathrm{mL}^{-1}$ $(\mathrm{p}<0.05$ versus infected lungs), from mechanically ventilated

Table 1.-Clinical and biochemical parameters of mechanically ventilated patients

\begin{tabular}{|c|c|c|c|c|c|}
\hline Patient type and No. & Sex & Age yrs & Duration of MV days & Site of infiltrate & BALF culture \\
\hline \multicolumn{6}{|l|}{ VAP patients } \\
\hline 1 & M & 51 & 11 & Left lower lobe & K. pneumoniae, E. coli \\
\hline 2 & M & 88 & 12 & Left lower lobe & Polymicrobial flora \\
\hline 3 & $\mathrm{~F}$ & 76 & 10 & Right middle lobe & P. aeruginosa \\
\hline 4 & $\mathrm{M}$ & 59 & 4 & Right middle lobe & Polymicrobial flora \\
\hline 5 & M & 75 & 6 & Left lower lobe & Polymicrobial flora \\
\hline \multicolumn{6}{|c|}{ Mechanically ventilated control patients } \\
\hline 1 & M & 73 & 6 & & \\
\hline 2 & M & 81 & 5 & & \\
\hline 3 & $\mathrm{~F}$ & 70 & 9 & & \\
\hline 4 & M & 56 & 7 & & \\
\hline 5 & M & 71 & 12 & & \\
\hline 6 & M & 33 & 8 & & \\
\hline 7 & M & 55 & 11 & & \\
\hline 8 & $\mathrm{~F}$ & 47 & 4 & & \\
\hline 9 & M & 23 & 6 & & \\
\hline 10 & $\mathrm{~F}$ & 49 & 8 & & \\
\hline
\end{tabular}

MV: mechanical ventilation; BALF: bronchoalveolar lavage fluid; VAP: ventilator-associated pneumonia; M: male; F: female; K. pneumoniae: Klebsiella pneumoniae; E. coli: Escherichia coli; P. aeruginosa; Pseudomonas aeruginosa. ${ }^{\#}$ : determined by chest radiograph. 

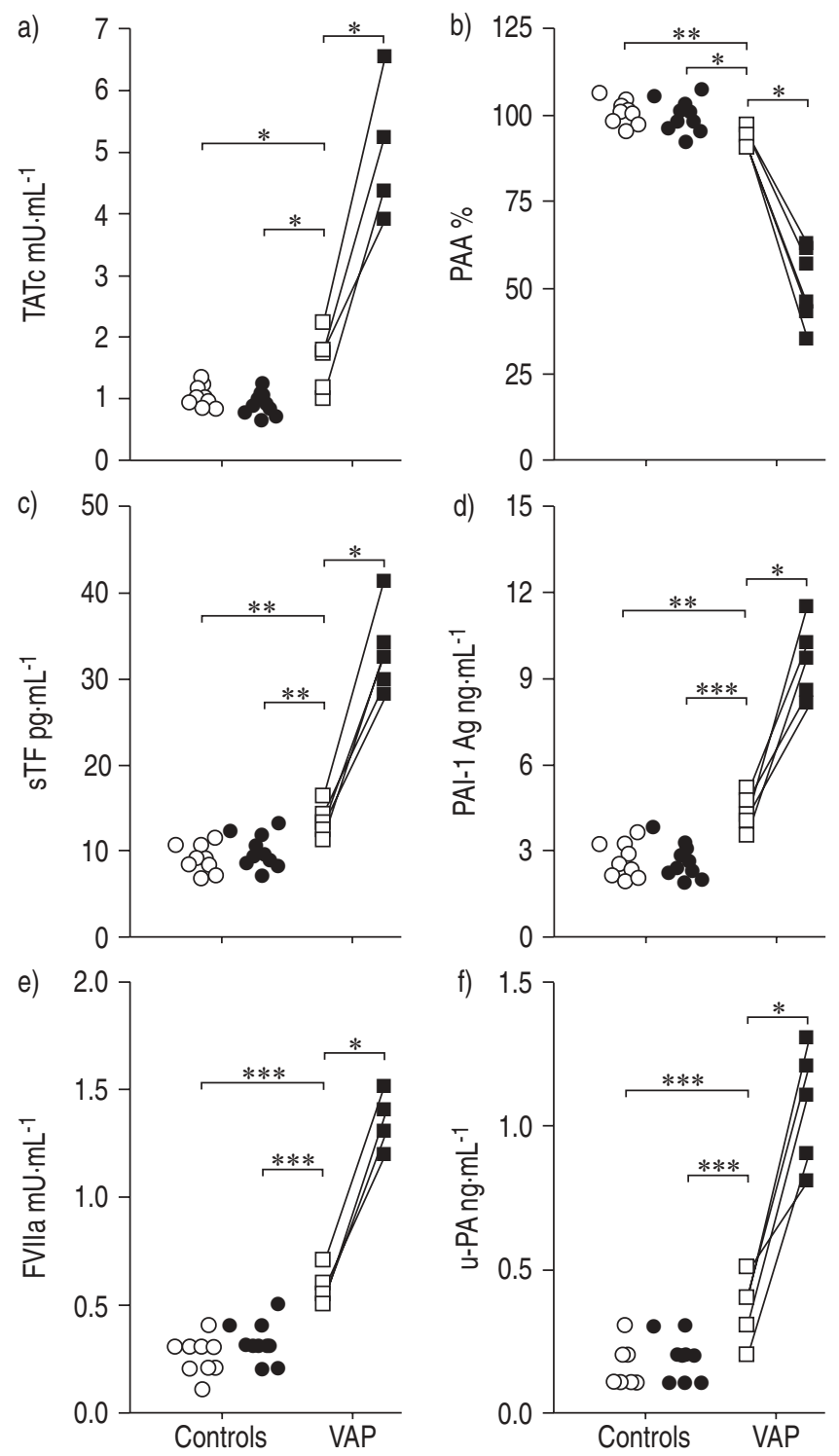

Fig. 1.- Levels of coagulation and fibrinolysis in bronchoalveolar lavage fluids (BALF) from healthy volunteers $(\bigcirc)$ and mechanically ventilated controls $(\bullet)$, and from the noninfected $(\square)$ and infected lungs (ם) in patients with ventilator-associated pneumonia (VAP). BALF concentrations of procoagulant markers are illustrated for a) thrombin-antithrombin complex (TATc), c) soluble tissue factor (sTF), and e) factor VIIa (FVIIa). BALF levels of fibrinolytic markers are illustrated for b) plasminogen activator activity (PAA), d) plasminogen activator inhibitor-1 antigen (PAI-1 Ag), and f) urokinase-type plasminogen activator (u-PA). Comparisons between infected and noninfected lungs (interconnected for the same patient) were performed with Wilcoxon signed-rank test for paired samples. Differences between VAP and control patients were analysed using the Mann-Whitney U-test. *: $\mathrm{p}<0.05 ; * *: \mathrm{p}<0.01$; ***: $\mathrm{p}<0.001$.

controls $153(75.0-314) \mathrm{pg} \cdot \mathrm{mL}^{-1}(\mathrm{p}=0.44$ versus infected lungs), and from healthy individuals 20.3 (16.7-73.4) $\mathrm{pg} \cdot \mathrm{mL}^{-1}(\mathrm{p}=0.05$ versus infected lungs $)$.

\section{Discussion}

In the present study, patients with VAP had a dramatic increase in local procoagulant activity and a simultaneous depression of fibrinolysis. The procoagulant shift of the haemostatic balance occurred in a compartmentalised fashion, i.e. disturbed fibrin turnover was restricted to the site of infection. Although the current study involved only five patients with unilateral VAP, the data derived from lavages of the infected site showed no overlap with that from lavages of the noninfected lung or control subjects. Whilst BALF obtained from the noninfected lung of patients with VAP displayed modest coagulation disturbances, compared with BALF from control patients, spill of BALF from the infected to the noninfected site was unlikely, since the two-sided BAL was consistently started with lavage of the noninfected lung. Hence, these data indicate that a unilateral VAP results in moderate coagulation disturbances in the noninfected lung, mirroring the more pronounced hypercoagulant state in the contralateral infected lung.

Mechanical ventilation by itself may initiate, or aggravate, pulmonary inflammation [6]. However, in mechanically ventilated patients without pneumonia, there was no activation of coagulation and inhibition of fibrinolysis, whilst being ventilated with the same mechanical ventilation mode and settings. Furthermore, similar changes in fibrin turnover have been found in nonmechanically ventilated patients with unilateral community-acquired pneumonia [4]. Therefore, the explanation that enhanced fibrin formation was triggered by infection, and not mechanical ventilation, is favoured.

The TF-FVIIa pathway is essential for activation of the blood coagulation system during systemic bacterial infection $[2,7]$. TF has also been implicated in the procoagulant response in the lungs, being present at multiple sites in the airways, including the vascular adventitia, epithelial cells and alveolar macrophages. Inhibition of the TF-FVIIa pathway completely prevented intrapulmonary fibrin deposition and diminished lung inflammation after intratracheal delivery of endotoxin in rats [8]. In addition, TF inhibition attenuated lung inflammation and injury in experimental endotoxaemia and bacteraemia [9]. In the current study, it was demonstrated that activation of the TF-FVIIa pathway was detected in patients with VAP at the site of the infection. These findings suggested that local activation of the TF-FVIIa pathway contributes to activation of coagulation at the site of the infection during VAP, and that inhibition of the TF-FVIIa pathway could potentially be beneficial to patients with pulmonary inflammatory disease.

There has been much interest in the interaction between coagulation and inflammation in the lung. Several reports have been published on disturbed fibrin formation in patients with VAP, community-acquired pneumonia and acute lung injury [3-5]. Pro-inflammatory cytokines increase the expression of TF, activating the coagulation cascade, whilst fibrin itself has been shown to enhance the inflammatory response [1]. The current authors chose to measure IL-6 and TNF- $\alpha$ because they play an important role in the activation of coagulation and attenuation of fibrinolysis, respectively, during sepsis [7]. However, in the present study, TNF- $\alpha$ levels in BALF were either not detectable or very low, and varied widely, as previously demonstrated in both developing VAP and established community-acquired pneumonia [10, 11]. Likewise, concentrations of IL-6 showed a large variation. Although differences between infected and noninfected lungs in patients with VAP were statistically significant, local IL-6 levels in the infected lungs were not different from levels in mechanically ventilated controls without pneumonia. Apparently, during pulmonary inflammation, IL- 6 and TNF- $\alpha$ are not the major determinants in the activation of coagulation and depression of fibrinolysis within the pulmonary compartment.

In conclusion, ventilator-associated pneumonia is characterised by a local procoagulant shift in the haemostatic balance of the airways. The imbalance is mainly restricted to the infected pulmonary compartment, and is the net result of 
increased tissue factor-factor VIIa-mediated coagulation activity, together with the inhibition of fibrinolysis, related to high plasminogen activator inhibitor type 1 levels. Future studies are warranted to examine whether inhibition of the hypercoagulant response in the lung during pneumonia has a beneficial effect.

\section{References}

1. Levi M, Schultz MJ, Rijneveld AW, van der Poll T. Bronchoalveolar coagulation and fibrinolysis in endotoxemia and pneumonia. Crit Care Med 2003; 31: Suppl. 4, S238S242.

2. Levi M, Ten Cate H. Disseminated intravascular coagulation. N Engl J Med 1999; 341: 586-592.

3. Schultz MJ, Millo JL, Levi M, et al. Local activation of coagulation and inhibition of fibrinolysis in the lung during ventilator-associated pneumonia. Thorax 2004; 59: 130-135.

4. Rijneveld AW, Florquin S, Bresser P, et al. Plasminogen activator inhibitor type-1 deficiency does not influence the outcome of murine pneumococcal pneumonia. Blood 2003; 102: 934-939.

5. Günther A, Mosavi P, Heinemann S, et al. Alveolar fibrin formation caused by enhanced procoagulant and depressed fibrinolytic capacities in severe pneumonia. Comparison with the acute respiratory distress syndrome. Am J Respir Crit Care Med 2000; 161: 454 462.

6. Ranieri VM, Suter PM, Tortorella C, et al. Effect of mechanical ventilation on inflammatory mediators in patients with acute respiratory distress syndrome: a randomized controlled trial. JAMA 1999; 282: 54-61.

7. van der Poll T, de Jonge E, Levi M. Regulatory role of cytokines in disseminated intravascular coagulation. Semin Thromb Hemost 2001; 27: 639-651.

8. Miller DL, Welty-Wolf K, Carraway MS, et al. Extrinsic coagulation blockade attenuates lung injury and proinflammatory cytokine release after intratracheal lipopolysaccharide. Am J Respir Cell Mol Biol 2002; 26: 650-658.

9. Welty-Wolf KE, Carraway MS, Miller DL, et al. Coagulation blockade prevents sepsis-induced respiratory and renal failure in baboons. Am J Respir Crit Care Med 2001; 164: 1988-1996.

10. Millo JL, Schultz MJ, Williams C, et al. Compartmentalized production of cytokines and cytokine inhibitors in ventilator-associated pneumonia. Intensive Care Med 2004; 30: 6874.

11. Dehoux MS, Boutten A, Ostinelli J, et al. Compartmentalized cytokine production within the human lung in unilateral pneumonia. Am J Respir Crit Care Med 1994; 150: $710-716$. 